INPLASY

PROTOCOL

To cite: Liu et al. A MetaAnalysis: Intervention Effect of Mind-Body Exercise on Relieving Cancer-related Fatigue in Breast Cancer Patients. Inplasy protocol 202130051. doi: 10.37766/inplasy2021.3.0051

Received: 15 March 2021

Published: 16 March 2021

Corresponding author: Jianghua Zhu

zjhwx2021@126.com

Author Affiliation: Physical Education Department of DongHua University

Support: None.

Review Stage at time of this submission: Data analysis.

Conflicts of interest:

None declared.

\section{A Meta-Analysis: Intervention Effect of Mind-Body Exercise on Relieving Cancer-related Fatigue in Breast Cancer Patients}

\author{
Liu, C1; Zheng, XH²; Chen, R³ Zhu, JH
}

Review question / Objective: 1: What effect does mind-Body Exercise exercise have on cancer-related fatigue of breast cancer? 2: Which type, cycle and duration is the best intervention effect for cancer-related fatigue in breast cancer? Condition being studied: Currently, there is a large literature showing that physical activity has a positive effect on cancerrelated fatigue in patients with breast cancer;However, only a few studies have shown a positive effect of mind-body exercise on cancer-related fatigue in breast cancer patients.Therefore, this study aims to further explore the effect of mind-body exercise on cancer-induced fatigue in breast cancer patients.

INPLASY registration number: This protocol was registered with the International Platform of Registered Systematic Review and Meta-Analysis Protocols (INPLASY) on 16 March 2021 and was last updated on 16 March 2021 (registration number INPLASY202130051).

\section{INTRODUCTION}

Review question / Objective: 1: What effect does mind-Body Exercise exercise have on cancer-related fatigue of breast cancer? 2: Which type, cycle and duration is the best intervention effect for cancerrelated fatigue in breast cancer?

Condition being studied: Currently, there is a large literature showing that physical activity has a positive effect on cancer- 
related fatigue in patients with breast cancer;However, only a few studies have shown a positive effect of mind-body exercise on cancer-related fatigue in breast cancer patients. Therefore, this study aims to further explore the effect of mind-body exercise on cancer-induced fatigue in breast cancer patients.

\section{METHODS}

Participant or population: In this study, we selected the breast cancer patients.

Intervention: In the study, we selected the article with the intervention by yoga, tai chi, qigong, and so on.

Comparator: The control group received routine care, health care, etc.

Study designs to be included: RCT.

Eligibility criteria: Patients who were $\geq 18$ year-old and have been pathologically diagnosed with breast cancer.

Information sources: The computer retrieved the databases including Pubmed, Web of Science, The Cochrane Library, Embase, CNKI, WanFang Data and SinoMed.

Main outcome(s): The outcomes about the cancer-related fatigue, including BFI, CRF, FSI, PFS-R, FACIT-F, EORTC QLQ-C30.

Quality assessment / Risk of bias analysis: Two reviewers will independently critically appraise each included study using the Cochrane Risk of Bias tool for randomised controlled trials (RCTs) and the Downs and Black checklist for non-RCTs. Disagreements will be resolved through discussion.

Strategy of data synthesis: The data processing software used Reviewer Manager 5.3. This meta-analysis strictly followed the PRISMA guidelines. P value and 12 were adopted for heterogeneity test. If there showed statistical heterogeneity between the results of the study $(12 \geq 50 \%, P$
$<0.10$ ), the random effects model would be used, otherwise the fixed effects model would be used. The data included in this paper were continuous data, whose confidence interval of effect size was SMD $=95 \%$, and was conducted sensitivity analyses by excluding individual papers one by one.

Subgroup analysis: Planned subgroup analyses will be:(A)exercise type. (B)exercise cycle. (C)exercise duration.

Sensitivity analysis: The review manger was used for sensitivity analysis of results with high heterogeneity.

Country(ies) involved: China.

Keywords: mind-body exercise; breast cancer; cancer-related fatigue; metaanalysis.

Contributions of each author:

Author 1 - Cong Liu.

Email: 1921111043@sus.edu.cn

Author 2 - Xinhu Zheng.

Email: 1821111059@sus.edu.cn

Author 3 - Rao Chen.

Email: 1921111059@sus.edu.cn

Author 4 - Jianghua Zhu.

Email: zjhwx2021@126.com 